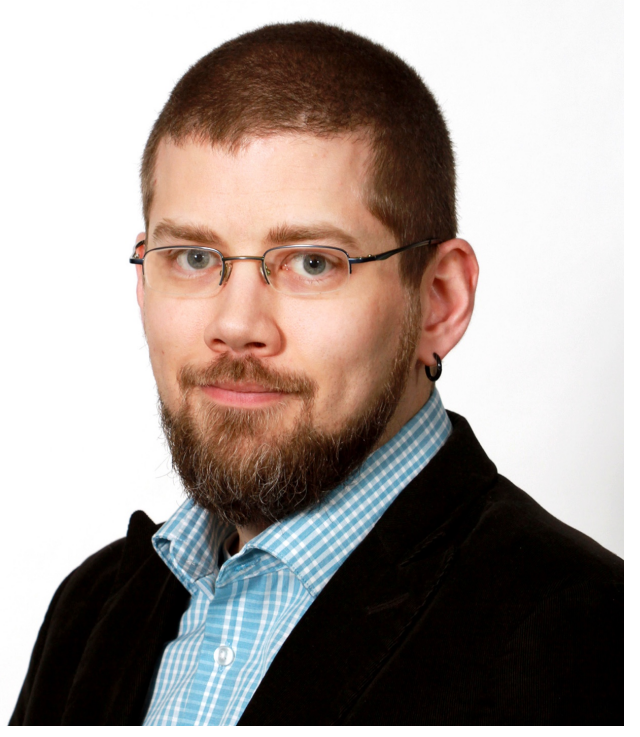

\title{
Western Faculty Profille: Dr. Dan Lizotte
}

\section{Emerald Liang ${ }^{1}$}

${ }^{1}$ Faculty of Schulich, Western University, London, Canada

No conflicts of interest declared

\section{Current research and day to day activities}

I have a Ph.D. in computer science and a Postdoctoral position studying statistics. I've always been interested in transforming data into actionable information, or decision support, for people whose jobs involve making decisions in a wide variety of scenarios. This interest is primarily focused on decision support in the healthcare world, but the things I work on can be applied to other fields as well.

People will have large, difficult-to-interpret data that they believe contains information useful to making everyday decision. However, it required a fair amount of processing, question formatting, and digesting to present it in a way that really helps them make the decision. I do almost no primary data collection, I work a lot with what I call "found data." Sometimes this data has been collected for research purposes, others times not. A large part of my data research experience is working with data sources that were not collected for research purposes, the biggest would be electronic medical record. These data files were not collected for research, but rather to help doctors with their patients.

\section{Since the data wasn't collected for research purposes, how do you deal with the variation in unstandardized information?}

[laughs] That's what really drives research in this area, not only for my colleagues and I, but also for other people who do similar things. We turn the question on its head and make dealing with the variation a research question in of itself, which is often the case for statisticians and computer scientists. When you reformulate it that way and you do methodological research, you develop methodology that allows you to use the data in the face of variation.

\section{Recognizing that not everyone who works with electronic medical records are researchers, how do you deal with incorrectly documented information?}

That's one of the reasons why I love being here at Western and why I love my job; I'm able to collaborate with end users [people who enter the enter the information into the database] who use the software and are interested in the same questions that I'm interested in. They can help me understand the different use patterns and therefore what the data actually means.

When you work on problems like this where you don't have that kind of connection with how the data is actually being created, there's a big danger of misinterpreting the data. If you can't get some insight from someone who actually creates the data, you can be lead astray and draw the wrong conclusion.

I'd also like to note really quickly that a lot of the software that people use on a daily basis is really bad, and it's not often no their fault that they make a mistake entering the data. I'm not surprised at that and I don't necessarily fault them for when it happens.

\section{How will this kind of research impact the future of medicine?}

I think it will give us a clearer, more comprehensive and richer picture, especially of patients who are currently under the care of doctors. 
When a doctor makes a decision about their patient, they're thinking about their training in medical school and about all of their past experiences. They are thinking about all the other patients in their career who are like this patient or not like this patient, and what happened to them when they had different kinds of treatment.

I think the way forward is to mirror that thought process and make these EMR (electronic medical record) data representative of the collective experience of doctors who have worked on them. It'll be transformational if we can make it represent useful information that can complement the training and expertise that doctors already have. If instead of having seen five patients, it's like the doctors has seen five million, that can really matter for rare diseases or rare events that the doctor might not have seen before.

\section{Would you then say that this use of technology is less of a complete take over and more complementary?}

My opinion is that the complementary route is the way to go, and that's why I talked about decision support in the beginning; how can we best support people as they do their jobs.

\section{In your research you did a lot of studies on reinforcement learning as part of machine learning and $\mathrm{Al}$ in general - could you talk a bit about that?}

Just briefly, when I describe reinforcement learning to someone, it's really just about using data to make good sequences of decisions rather than using data to make a single good decision right now. When people have done reinforcement learning research over the past twenty - thirty years, they have always wanted to build something awesome like R2D2. They want to build an entity that acts on the world and achieve long term goals

For me, the progression has most recently been about improving our ability to predict the outcomes of long sequences of actions. That's what helped reinforcement learning take a prominent role in terms of playing games like go and checkers. I'm hoping the same advances can lead to improvement in chronic disease treatment, where there's an inherent sequential decisionmaking aspect. For diseases that we can't cure, there's some collection of diagnosis treatment and monitoring that's happening to the same person over time. The same methods that are used for long term decision making in other areas should apply in these cases too.

\section{What are some of the unforeseen joys in your research career?}

The unforeseen joys for me have been the people that I met and been able to work with: students, other faculty members, medical health professionals, and community partners. People who are willing to give me their ear and talk about the potential for these kinds of methods, who are willing to help me interpret their data and help me understand how the data was created so that we can ensure that whatever we produce is meaningful. Even going through the steps required to share the data is something that I really appreciate. One of the best things is the number of people l've found who were excited about the potential for this research and its application in different fields.

\section{What about the unforeseen difficulties experienced?

that \\ you've}

I think a difficulty I underestimated was the ability to find collaborator if you're not in an environment that really fosters that. Western does foster collaboration, it just took me a while to get to a place where I was able to connect with those people.

One of the biggest challenges I had before I got to Western was finding those key collaborators. I can do methodological research on my own, but I'm much happier in collaborative projects. That was a challenge, but l'm very happy to be here now to make those connections

\section{Do you have any advice for undergraduate students who don't have these connections yet?}

I think the time is right for interdisciplinary education and interdisciplinary research. My only advice for students is to avoid getting trapped in too narrow a view of what they're interested in and what they want to accomplish in the future. Such as when they're picking their courses. Sometimes there's not a lot of flexibility for students I understand that, but I would really encourage students not to discount courses that may seem a bit unusual or a bit out there or different from their main path. I think that can be a really valuable thing.

\section{As a cross-appointed professor, how do you balance the two separate fields?}

It's hard. When I look at projects I try to think "is this more heavily on the computer science side or is it more heavily on the epidemiology side?" Then when I'm thinking about finding students to work on it, l'll look in one department versus the other department depending on whether I think it's really crucial that the person working on this has in-depth computing skills or whether it's really key that the person understands causal inference and epidemiological concepts. I keep it balanced on a project by project basis, thinking about who are the students and what do they need to really succeed on this project. 
To Learn more on Dr. Lizotte's lab and research, please visit his website at:

http://www.csd.uwo.ca/ dlizotte 